\title{
Intraoperative Sentinel Lymph Node Evaluation: Implications of Cytokeratin 19 Expression for the Adoption of OSNA in Oral Squamous Cell Carcinoma
}

\author{
Richard Shaw, MD, FRCS, FDS ${ }^{1}$, Anders Christensen, MD², Kapil Java, MRCS ${ }^{1}$, Rehab El Maddani, BDS, MRes ${ }^{1}$, \\ Triantafillos Liloglou, BSc, PhD ${ }^{1}$, Triantafyllou Asterios, PhD, FRCPath ${ }^{3}$, Christian von Buchwald, MD, DMSc ${ }^{2}$, \\ Irene Wessel, MD, $\mathrm{PhD}^{2}$, Katalin Kiss, $\mathrm{MD}^{4}$, Andreas Kjaer, MD, PhD, DMSc ${ }^{5}$, Giedrius Lelkaitis, $\mathrm{MD}^{4}$, \\ Anna Long, BSc, MSc ${ }^{6}$, Janet Risk, BSc, PhD $^{1}$, and Max Robinson, PhD $^{7}$ \\ ${ }^{1}$ Department of Molecular Clinical Cancer Medicine, Mersey Head \& Neck Oncology Group, University of Liverpool, \\ Liverpool, UK; ${ }^{2}$ Department of Otolaryngology, Head \& Neck Surgery and Audiology, Rigshospitalet, Copenhagen \\ University Hospital, Copenhagen, Denmark; ${ }^{3}$ Cellular Pathology, Aintree University Hospitals NHS Foundation Trust, \\ Liverpool, UK; ${ }^{4}$ Department of Pathology, Rigshospitalet, Copenhagen University Hospital, Copenhagen, Denmark; \\ ${ }^{5}$ Department of Clinical Physiology, Nuclear Medicine \& PET and Cluster for Molecular Imaging, Rigshospitalet, \\ Copenhagen University Hospital, Copenhagen, Denmark; ${ }^{6}$ Cellular Pathology, Newcastle upon Tyne Hospitals \\ NHS Foundation Trust, Newcastle upon Tyne, UK; ${ }^{7}$ Centre for Oral Health Research, Newcastle University, \\ Newcastle upon Tyne, UK
}

\begin{abstract}
Background. Intraoperative analysis of sentinel lymph nodes would enhance the care of early-stage oral squamous cell carcinoma (OSCC). We determined the frequency and extent of cytokeratin 19 (CK19) expression in OSCC primary tumours and surrounding tissues to explore the feasibility of a "clinic-ready" intraoperative diagnostic test (one step nucleic acid amplification-OSNA, sysmex).

Methods. Two cohorts were assembled: cohort 1, OSCC with stage and site that closely match cases suitable for sentinel lymph node biopsy (SLNB); cohort 2, HNSCC with sufficient fresh tumour tissue available for the OSNA assay (>50 mg). CK19 assays included qRT-PCR, RNA
\end{abstract}

Richard Shaw and Anders Christensen are joint first authors. Janet Risk and Max Robinson are joint senior authors.

Electronic supplementary material The online version of this article (doi:10.1245/s10434-016-5337-6) contains supplementary material, which is available to authorized users.

(C) The Author(s) 2016. This article is published with open access at Springerlink.com

First Received: 26 March 2016;

Published Online: 8 July 2016

R. Shaw, MD, FRCS, FDS

e-mail: rjshaw@liv.ac.uk in situ hybridisation (ISH), and immunohistochemistry (IHC), as well as OSNA.

Results. CK19 mRNA expression was detected with variable sensitivity, depending on method, in $60-80 \%$ of primary OSCC tumours, while protein expression was observed in only $50 \%$ of tumours. Discordance between different techniques indicated that OSNA was more sensitive than qRTPCR or RNA-ISH, which in turn were more sensitive than IHC. OSNA results showed CK19 expression in $80 \%$ of primary cases, so if used for diagnosis of lymph node metastasis would lead to a false-negative result in $20 \%$ of patients with cervical lymph node metastases.

Conclusions. OSNA in its current form is not suitable for use in OSCC SLNB due to inadequate expression of the CK19 target in all case. However, the same assay technology would likely be very promising if applied using a more ubiquitous squamous epithelial target.

Renewed interest in sentinel lymph node biopsy (SLNB) for early-stage oral squamous cell carcinoma (OSCC) has resulted from reassuring data with $95 \%$ negative predictive value (NPV) and also recent trials reinforcing the survival benefit of surgical neck staging. ${ }^{1-3}$ A significant drawback of SLNB is that, in the event of a positive lymph node, a costly (and more morbid) second surgical episode is necessitated. This delay, mandated by serial examination of 
SLN, delays the commencement of adjuvant therapy and creates additional patient distress. SLNB in OSCC would be facilitated by intraoperative staging; however, frozensection analysis has been found to be somewhat insensitive, certainly highly operator dependent, and has not found general acceptance. ${ }^{4-6}$ PCR-based techniques have been reported for head and neck squamous cell carcinoma (HNSCC) but lack a "clinic-ready" platform. ${ }^{7,8}$

One-step nucleic acid amplification (OSNA) uses loopmediated isothermal amplification (LAMP), amplifying RNA with high sensitivity, specificity, efficiency, and rapidity under isothermal conditions. ${ }^{9}$ OSNA employs six specially designed primers at eight sequences within $C K 19$ mRNA subtending high sensitivity and specificity. In breast cancer, OSNA has been validated to at least $96 \%$ concordance with histopathology and has been widely adopted and approved in UK NICE guidelines. ${ }^{10,11}$ OSNA necessitates an additional 30-40 min operative time but avoids second surgeries and accelerates commencement of adjuvant therapies from 8.4 to 6.2 weeks. $^{12}$

In HNSCC, the clinical potential of OSNA is unproven, and careful validation is required. Although gene signatures for OSCC or epithelial tissue have been developed with sensitive RT-PCR using other target cytokeratins, or PVA/ EPCAM, the opportunity around $C K 19$ is the availability of a "clinic-ready" diagnostic test with stringent quality assurance. $^{7,8}$ Several reports have shown that CK19 is a component of the cytoskeleton of HNSCC and qRT-PCR for cytokeratins appear sensitive and specific in detecting cervical lymph node metastasis in HNSCC. ${ }^{8,13-15}$ CK19 OSNA has been validated recently for lymph node staging in colorectal and stomach adenocarcinoma. ${ }^{16-19}$ The extent of CK19 expression in HNSCC, and therefore whether OSNA could have clinical utility, remains unproven.

Goda et al. analysed 213 HNSCC lymph nodes with CK19 OSNA and suggested an overall accuracy of 94\% per node and $94 \%$ per patient. ${ }^{20}$ Matsuzuka et al. found a NPV of $95.9 \%$ in HNSCC. ${ }^{21}$ Suzuki examined CK19 expression in HSNCC, finding a lower rate of expression and suggesting that clinical use of OSNA only in a selected subset of HNSCC known to be CK19 positive. ${ }^{22}$ All three studies were undertaken in a Japanese population with a variety of stages and sites of HNSCC. For example, Goda et al. report on cT1-4 and N0-3 OSCC and Matsuaka et al. report on a combination of HNSCC sites and also include advanced stages. ${ }^{20,21}$ Because SLNB is routinely offered only to cT12N0 OSCC, these reports do not ideally reflect the target clinical population in question. It remains uncertain if the expression of CK19 is sufficiently high and uniform to make the CK19 OSNA suitable for use in OSCC SLNB.

The purpose of this study was to establish the frequency and extent of CK19 expression in primary OSCC and surrounding, potentially contaminating, tissues. We established expression of $C K 19$ mRNA by using both OSNA and other techniques, as well as protein expression. In the event that CK19 expression is $<95 \%$, we will pilot assays to be used on diagnostic biopsies of primary tumours to stratify them as suitable, or not, for OSNA analysis of SLNB. Lastly, we tested the concordance between matched primary tumour and metastatic lymph node in CK19 expression.

\section{METHODS}

\section{Tissue}

A clinical cohort was assembled from the tissue banks of the Universities of Liverpool and Copenhagen with appropriate ethical approvals and consent. Clinicopathological characteristics and results are summarised in Supplemental Table S1.

Cohort 1 (43 cases from Liverpool) met the criteria: OSCC, clinical stage cT1N0 and cT2N0, formalin-fixed paraffin-embedded (FFPE) and fresh-frozen tumour tissue available. The OSNA assay interrogates fresh (or frozen) sentinel lymph nodes $>50 \mathrm{mg}$, preferably in their entirety. This presented an ethical and logistic barrier as the lymph nodes are required for histopathological staging, and banked primary tumour samples were exclusively $<50 \mathrm{mg}$ in T1/T2 OSCC. We therefore elected to analyse primary tumour to establish CK19 expression using a number of assays, excluding OSNA. Thirty-four of 43 had available matched FFPE lymph nodes.

Cohort 2 (87 cases: 44 Liverpool and 43 Copenhagen) met the criteria: OSCC, $>50 \mathrm{mg}$ snap-frozen primary tumour tissue, most of these were cT3/4 cases.

\section{Tissue Preparation and Handling}

Cohort 1. Immunohistochemistry (IHC): $4-\mu \mathrm{m}$ sections were stained for CK19 protein by two methods: a mouse monoclonal antibody (clone b170, Leica Biosystems) on a Ventana Benchmark Ultra Autostainer (Ventana Medical Systems, Inc.) at a dilution of 1:100 using standard retrieval conditions (MMC1) and the detection polymer Ultraview (Ventana Medical Systems, Inc.): a mouse monoclonal antibody (clone RCK 108, Dako) diluted 1:100 and the EnVision FLEX system on an Autostainer Link 48 instrument (Dako) using high $\mathrm{pH}$ antigen retrieval. Negative controls omitted addition of the primary antibody.

In situ hybridization (ISH): CK19RNA ISH was performed on 4- $\mu$ m FFPE sections using proprietary reagents (RNAscope, Advanced Cell Diagnostics, Inc.). Sections were deparaffinised and pretreated with heat and protease before hybridisation with target-specific probes: CK19, PPIB (constitutively expressed endogenous gene; positive control) and dapB (bacterial mRNA; negative control) in a dedicated hybridization oven (HybEZ oven, Advanced Cell 
FIG. 1 A primary tumour that shows weak, heterogeneous CK19 positivity, and a corresponding subcapsular lymph node metastasis with stronger CK19 staining. This case illustrates the difficulty, with either IHC or ISH, to offer a confident diagnostic test to identify cases from diagnostic biopsy suitable for CK19 OSNA

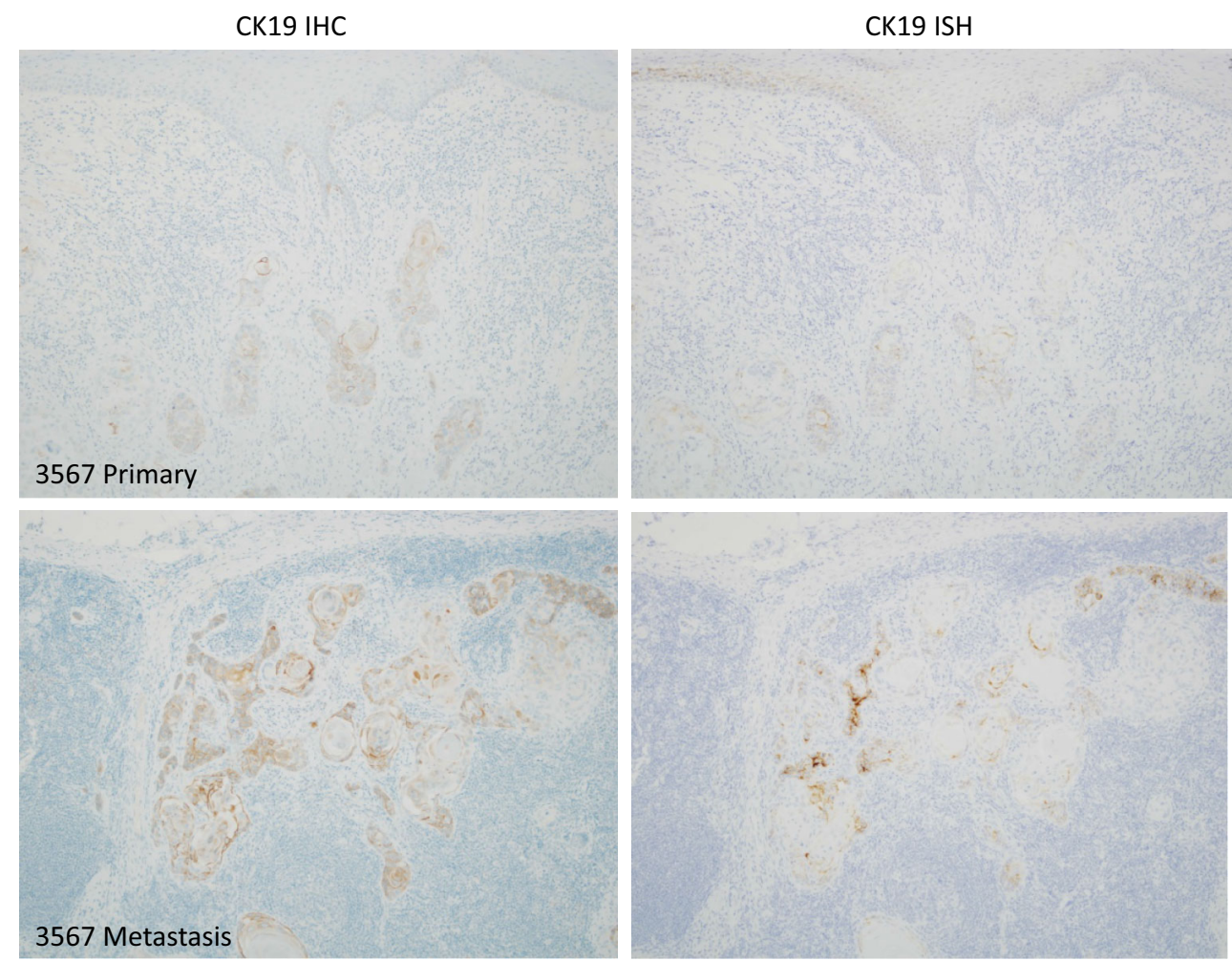

Diagnostics, Inc.). Probe hybridization was detected using the chromogen 3,3'-diaminobenzidine (DAB).

Both IHC and ISH techniques were optimized using known positive (breast ductal carcinoma) and negative tissue (lymph node). Tissue cores from controls constituted a "control block" - sections of which were mounted on each test slide to ensure quality staining methods. The tests were scored by two pathologists (MR \& AT). Staining was assessed by assigning an intensity score ( 0 , no staining; 1 , weak; 2 , moderate; 3 , strong) and percentage of malignant cells stained. These were used to calculate an $\mathrm{H}$ score (product of intensity and percentage) but also classified in a binary fashion (positive vs. negative).

RNA was prepared from fresh-frozen tissue of primary tumours using an miRNeasy kit (Qiagen), and following reverse transcription (cDNA kit, Applied Biosystems), a CK19 qRT-PCR assay was performed with the following primers/probe; Fwd: 5'CACTACTACACGACCATCC AGGAC 3', Rev: 5' CGGAAGTCATCTGCAGCCA 3', Probe: $5^{\prime}$ TAMRA-ACGGGCATTGTCGATCTGCAG GAC-BHQ2. The qPCR reaction utilised the Universal Master Mix II (Applied Biosystems), the thermal profile: $50{ }^{\circ} \mathrm{C}$ for $2 \mathrm{~min}, 95^{\circ} \mathrm{C}$ for $10 \mathrm{~min}, 45$ cycles of $95^{\circ} \mathrm{C}$ for $15 \mathrm{sec}$, and $60{ }^{\circ} \mathrm{C}$ for $1 \mathrm{~min}$, using a $7500 \mathrm{FAST}$ instrument (Applied Biosystems). The relative quantification (RQ)

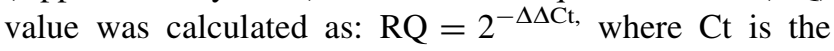
cycle threshold for each target.

Cohort 2. OSNA: OSCC biopsies from cohort 2 with mass between 50 and $600 \mathrm{mg}$ were snap frozen and stored at $-80{ }^{\circ} \mathrm{C}$ until shipment to Sysmex on dry ice. Samples were processed according to manufacturer's instructions (Sysmex, Kobe, Japan) using a designated instrument (RD$100 i$ ) and reagent system (LYNOAMP \& LYNORHAG). Individual tumour samples were placed in $4 \mathrm{ml}$ of homogenizing buffer LYNORHAG $(0.2 \mathrm{M}$ glycine- $\mathrm{HCl}$ pH 3.5, 5\% Brij35 and 20\% DMSO), and homogenised for $60 \mathrm{~s}$ at $10,000 \mathrm{rpm}$ with a Polytron System PT1300D (Kinematica AG, Switzerland) and LYNOPREP blades to prepare a homogeneous lysate. One milliliter of lysate was centrifuged to remove cell debris and then further diluted 1:10 and 1:100 with LYNORHAG. The diluted lysates were used directly for amplification without RNA extraction or purification. Isothermal amplification reactions were performed at $65^{\circ} \mathrm{C}$. The rise time required for precipitation of magnesium pyrophosphate to reach a turbidity of $0.1 \mathrm{OD}$ at $465 \mathrm{~nm}$ was obtained for each sample and the number of CK19 mRNA copies determined using a calibration curve. OSNA was classified as following: $(-)=<250$ copies; $(-\mathrm{L})=<250$ copies; $(+)=>250$ and $<5000$ copies, $(++)=>5000$ copies; $(++)$ or $(+)$ were positive results, whereas $(-)$ or $(-\mathrm{L})$ were negative.

RNA quality was analysed for negative (- or $-\mathrm{L})$ samples to exclude false negatives. OSNA lysates were processed with the Qiagen RNeasy kit (Qiagen, Venlo, Netherlands). Total RNA was quantified spectrophotometrically (260/280 nm ratio). RNA integrity was assessed using RNA Integrity Number (RIN) with a Bioanalyser (Agilent, Santa Clara, CA). 
TABLE 1 Test results for primary tumours with corresponding lymph node metastases

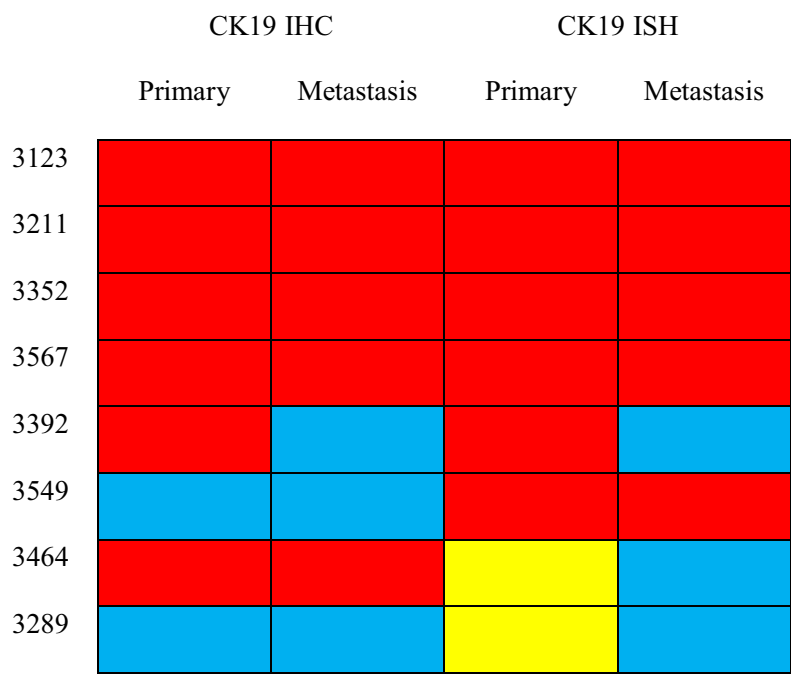

Red positive, blue negative, yellow not available, test failed quality assurance checks
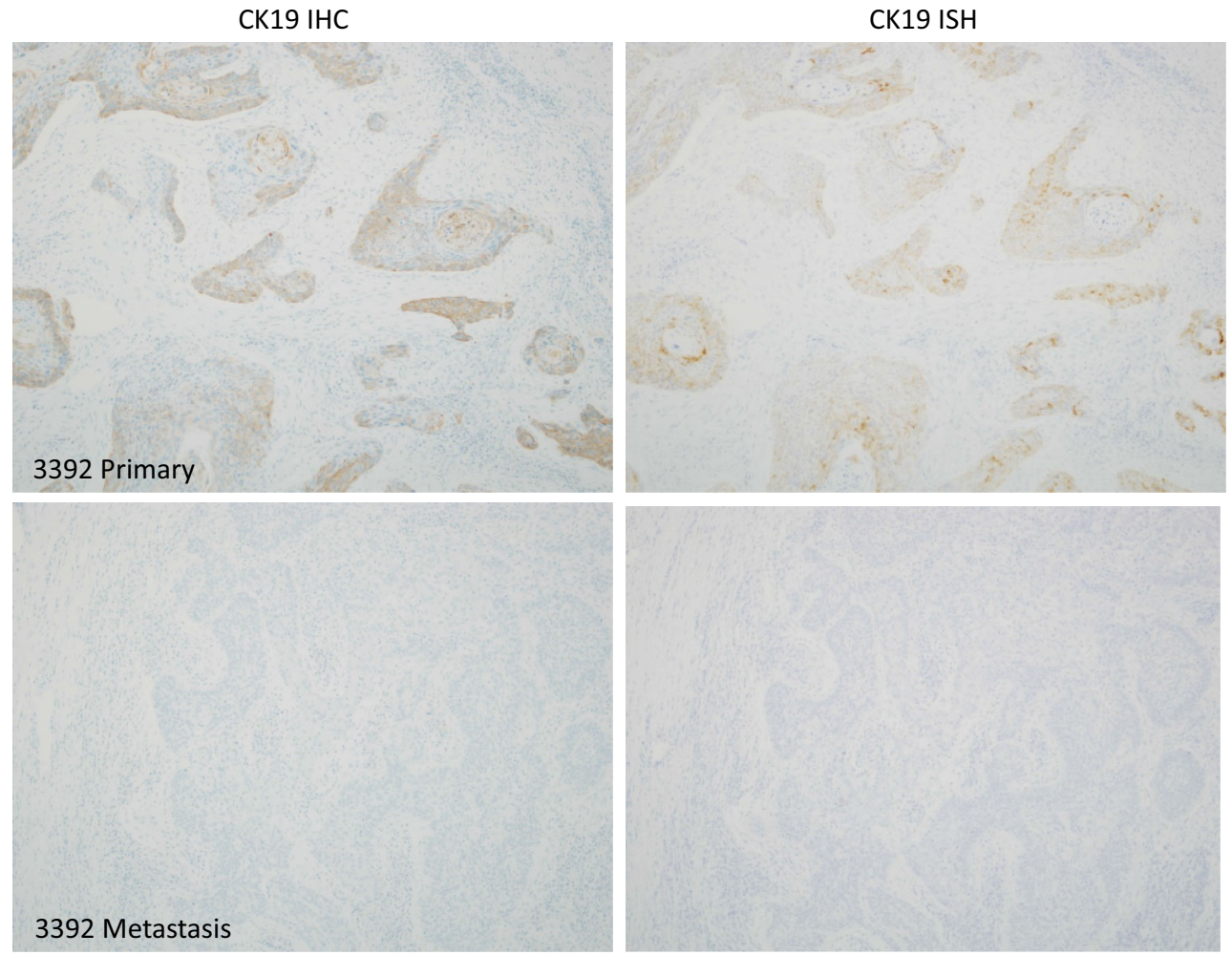

FIG. 2 A primary tumour that shows weak, heterogeneous CK19 positivity, and a corresponding lymph node metastasis with no CK19 staining. If this case had undergone SLNB analysis using CK19

RNA was prepared from unused OSNA lysates and from a separate aliquot of fresh-frozen tissue from the same tumours, reverse transcribed and subject to CK19 qRTPCR assay as described above.
OSNA, even with the apparent security of a "positive" primary tumour, it is likely that a false-negative result would be returned with consequent undertreatment and neck recurrence

Interplate qRT-PCR variation was reduced by using the $\Delta \Delta \mathrm{Ct}$ method to normalise expression with respect to two tumours that had previously been shown to highly express CK19. A technical threshold of $0.005 \times$ the mean $\Delta \mathrm{Ct}$ of 
TABLE 2 qRT-PCR concordance with IHC and ISH staining

\begin{tabular}{lrrrrrr}
\hline & & \multicolumn{2}{c}{ IHC } & & \multicolumn{2}{c}{ ISH } \\
\cline { 3 - 4 } \cline { 5 - 6 } & & + & - & & + & - \\
\hline qRT-PCR & + & 11 & 7 & 11 & 4 \\
qRT-PCR & - & 3 & 5 & 5 & 2 \\
\hline
\end{tabular}

the reference tumours was observed in two experiments and was adopted to distinguish positive from negative CK19 expression in all qRT-PCR experiments.

\section{RESULTS}

Cohort 1 . Of 43 primary cT1/T2N0 tumours tested with CK19 IHC, 21 (48.8\%) were positive. Twenty-nine of 39 primary tumours evaluable in $C K 19$ RNA ISH tests were CK19 positive (74.4\%); 4 failed quality assurance checks. CK19 IHC was concordant with the CK19 RNA ISH in 26 of 39 cases $(66.7 \%)$. For both tests, the staining was generally weak and heterogeneous (Figs. 1,2) with positive cases having $\mathrm{H}$ scores between 5 to 200. Discordant cases ( $n=13$ ) had lower H scores (mean 42.7; range 5-160). Of the 13 discordant results, 9 were positive in RNA ISH and negative in IHC, reflecting higher sensitivity of RNA ISH. Stage I/II OSCC was less likely to be positive by IHC than stage III/IV $(P<0.01)$. No such discrepancy was observed for RNA ISH.

CK19 IHC and CK19 RNA ISH were concordant in 6 of 8 cases with corresponding lymph node metastases, omitting two failed tests (Table 1). In one case, the primary tumour was positive for both tests, but the corresponding lymph node metastasis was negative (patient 3392; Fig. 2; Table 1). Lymph nodes with no evidence of metastatic carcinoma $(n=26)$ did not contain any CK19-positive cells. There were no epithelial lymph node inclusions (salivary or thyroid); however, in one case CK19 positive perinodal salivary gland tissue was included in the section, but this might have been dissected free prior to analysis in an SLNB protocol.

Eighteen of $26(69 \%)$ primary cT1/T2N0 tumour tissues were positive for $C K 19$ mRNA by qRT-PCR. CK19 qRTPCR was concordant with IHC in 16 of 26 cases (62\%) and with CK19 RNA ISH in 13 of 22 cases (59\%; Table 2). Discordant IHC cases tended to be positive by qRT-PCR (7/10). By contrast, discordant ISH cases were equally likely to be positive or negative (4/9 positive by qRTPCR); however, the ISH positives had lower $\mathrm{H}$ scores (mean 33.0, range 5-160).

Cohort 2. Of 87 primary tumour samples analysed by OSNA (Table 3), 7 were excluded because of compromised RNA integrity (low RIN). Examination of representative, H\&E stained sections from the 43 Danish
TABLE 3 Distribution of CK19 mRNA expression by OSNA

\begin{tabular}{lll}
\hline OSNA & No. of samples $(\%)$ & $\%$ \\
\hline$(++)$ & $45(56)$ & $65(81)$ \\
$(+)$ & $20(25)$ & \\
$(-)$ & $12(15)$ & \\
$(-\mathrm{L})$ & $3(4)$ & \\
Total & $80(100)$ &
\end{tabular}

samples identified 5 that did not contain tumour tissue by pathological examination and 1 with compromised RNA integrity. The remaining 4 were OSNA positive: 2 contained oral epithelium and 2 contained salivary tissue. Of 76 tumour samples, 61 (80\%) were CK19 mRNA positive by OSNA, with no correlation for either tumour stage or site (Supplementary Table S2).

Thirty-nine of the tumours from Liverpool had sufficient tissue to allow extraction of mRNA from a separate portion of the tumour. Of these, $23(59 \%)$ were CK19 positive. qRT-PCR data was concordant with OSNA data in 29 of 37 (78\%) of cases, with OSNA proving the most sensitive test in all 8 discordant cases. To investigate this more fully, RNA from the OSNA tissue lysates from all 87 samples was subject to qRT-PCR. Four samples were excluded based on the low RNA levels (GAPDH amplification). Of these samples, 56 of $83(67 \%)$ were positive for $C K 19$ expression, showing concordance with OSNA data in 70 of $81(85 \%)$ of cases. All discordant cases demonstrated positivity by OSNA but were negative by qRT-PCR.

\section{CONCLUSIONS}

CK19 expression is detectable for $50-80 \%$ of OSCC, depending upon the assay used. RNA-ISH and qRT-PCR are more sensitive than IHC, whereas OSNA appears to be the most sensitive method. The prevalence of CK19 expression by OSNA is still, at $80 \%$, insufficient to suggest that OSNA could be used without prior screening of biopsy tissue for CK19, because it could result in $20 \%$ of positive lymph nodes being called as false-negative. CK19 expression in OSCC has been reported previously to range from $53 \%$ to $91 \% .{ }^{13,14}$ Our results confirm that CK19 can be detected only in a subset of primary tumours. In this regard, OSCC differs from breast, colorectal and stomach sites, all adenocarcinomas, where CK19 OSNA has been clinically validated. Although the chemistry and platform available through OSNA appear to be well suited to clinical use in being highly reliable, sensitive, and specific, the gene target CK19 appears to offer insufficient expression in OSCC for clinical application. Should a more appropriate gene target (perhaps CK5 or 14) be available, it may be that this would be suitable, subject to the appropriate and necessary clinical validations. 
Although, theoretically, OSNA might be used on a fresh biopsy sample to select CK19 positive tumours suitable for OSNA assay in SLNB, concern remains that surrounding oral mucosa or salivary gland could be included leading to a false positive. CK19 mRNA ISH performed on an existing FFPE diagnostic biopsy might be more convenient and provide histological context, avoiding false positives. However, our results show that CK19 mRNA ISH expression was usually low and heterogeneous, limiting diagnostic confidence and making the assay vulnerable to interobserver variability. Consequently, we could not suggest a reliable assay to stratify which tumours are suitable for OSNA assay in SLNB.

In one case, the primary tumour was positive and the matched lymph node metastasis was negative by both CK19 ISH and IHC. Contamination in the neck structures with ectopic salivary $(0.9 \%)$ or thyroid tissue $(1.5 \%)$ have been reported either within or immediately surrounding lymph nodes and could produce false positives in any methodology that uses solid specimens. ${ }^{23,24}$ It may be that careful dissection of single SLNB would eliminate this, but again a validation study would be helpful.

Our data successfully incorporated a new assay (CK19 mRNA ISH) and shows potential clinical avenues in OSCC for molecular diagnostics. We have CK19 data on 123 OSCC that effectively rules out the need for potentially burdensome, and clinically risky, validation studies. The international collaboration between two academic head and neck cancer centres and industry augers well should a more suitable assay become available. Such an assay might additionally be applicable to cutaneous SCC and anogenital SCC, which would increase the test's commercial viability. It is encouraging that OSNA assays with differing gene targets, most recently with MMP7 (matrix-metalloproteinase 7) are available. ${ }^{25}$

The concept of intraoperative diagnostics in OSCC remains attractive but awaits a suitable assay. At present, SLNB analysis is based on evaluation of stepped serial sections from only a proportion of the sentinel node, thus a rapid technique examining the entire sentinel node for tumour deposits may provide more accurate staging. An automated intraoperative method also would avoid the substantial additional workload for the pathology team performing serial SLNB examination. In head and neck oncology, intraoperative diagnostics appear even more attractive than in melanoma and breast, because OSCC remains largely a surgically treated disease and completing all surgery in one operation would facilitate the wider acceptance of SLNB.

ACKNOWLEDGMENTS The authors thank Monika Zaenkert, Gabriele Ebling, and Franziska Duerigen, Sysmex Europe GmbH, Germany.
OPEN ACCESS This article is distributed under the terms of the Creative Commons Attribution 4.0 International License (http:// creativecommons.org/licenses/by/4.0/), which permits unrestricted use, distribution, and reproduction in any medium, provided you give appropriate credit to the original author(s) and the source, provide a link to the Creative Commons license, and indicate if changes were made.

\section{REFERENCES}

1. 1. Pedersen NJ, Jensen DH, Hedback N, et al. Staging of early lymph node metastases with the sentinel lymph node technique and predictive factors in $\mathrm{T} 1 / \mathrm{T} 2$ oral cavity cancer: a retrospective single-center study. Head Neck. 2016;38:1033-40.

2. Schilling C, Stoeckli SJ, Haerle SK, et al. Sentinel European Node Trial (SENT): 3-year results of sentinel node biopsy in oral cancer. Eur J Cancer. 2015;51(18):2777-84.

3. D'Cruz AK, Vaish R, Kapre N, et al. Elective versus therapeutic neck dissection in node-negative oral cancer. $N$ Engl $\mathrm{J}$ Med. 2015;373(6):521-9.

4. Civantos FJ, Moffat FL, Goodwin WJ. Lymphatic mapping and sentinel lymphadenectomy for 106 head and neck lesions: contrasts between oral cavity and cutaneous malignancy. Laryngoscope. 2006;112(3 Pt 2 Suppl 109):1-15.

5. Tschopp L, Nuyens M, Stauffer E, Krause T, Zbaren P. The value of frozen section analysis of the sentinel lymph node in clinically N0 squamous cell carcinoma of the oral cavity and oropharynx. Otolaryngol Head Neck Surg. 2005;132(1):99-102.

6. Alkureishi LW, Burak Z, Alvarez JA, et al. Joint practice guidelines for radionuclide lymphoscintigraphy for sentinel node localization in oral/oropharyngeal squamous cell carcinoma. Ann Surg Oncol. 2009;16(11):3190-210.

7. Ferris RL, Xi L, Seethala RR, et al. Intraoperative qRT-PCR for detection of lymph node metastasis in head and neck cancer. Clin Cancer Res. 2011;17(7):1858-66.

8. Garrel R, Dromard M, Costes V, et al. The diagnostic accuracy of reverse transcription-PCR quantification of cytokeratin mRNA in the detection of sentinel lymph node invasion in oral and oropharyngeal squamous cell carcinoma: a comparison with immunohistochemistry. Clin Cancer Res. 2006;12(8):2498-505.

9. Notomi T, Okayama H, Masubuchi $\mathrm{H}$, et al. Loop-mediated isothermal amplification of DNA. Nucl Acids Res. 2000;28(12):E63.

10. Snook KL, Layer GT, Jackson PA, et al. Multicentre evaluation of intraoperative molecular analysis of sentinel lymph nodes in breast carcinoma. Br J Surg. 2011;98(4):527-35.

11. NICE. Intraoperative tests (RD 100i OSNA system and Metasin test) for detecting sentinel lymph node metastases in breast cancer. London:NICE; 2013.

12. Klingler $S$, Marchal F, Rauch $P$, et al. Using one-step nucleic acid amplification (OSNA) for intraoperative detection of lymph node metastasis in breast cancer patients avoids second surgery and accelerates initiation of adjuvant therapy. Ann Oncol. 2013;24(9):2305-9.

13. Yamauchi K, Fujioka Y, Kogashiwa Y, Kohno N. Quantitative expression study of four cytokeratins and p63 in squamous cell carcinoma of the tongue: suitability for sentinel node navigation surgery using one-step nucleic acid amplification. J Clin Pathol. 2011;64(10):875-9.

14. Zhong LP, Chen WT, Zhang CP, Zhang ZY. Increased CK19 expression correlated with pathologic differentiation grade and prognosis in oral squamous cell carcinoma patients. Oral Surg Oral Med Oral Pathol Oral Radiol Endod. 2007;104(3):377-84.

15. Shores CG, Yin X, Funkhouser W, Yarbrough W. Clinical evaluation of a new molecular method for detection of 
micrometastases in head and neck squamous cell carcinoma. Arch Otolaryngol Head Neck Surg. 2004;130(8):937-42.

16. Croner RS, Schellerer V, Demund H, et al. One step nucleic acid amplification (OSNA) - a new method for lymph node staging in colorectal carcinomas. J Trans Med. 2010;8:83.

17. Yamamoto H, Sekimoto M, Oya M, et al. OSNA-based novel molecular testing for lymph node metastases in colorectal cancer patients: results from a multicenter clinical performance study in Japan. Ann Surg Oncol. 2011;18(7):1891-8.

18. Kumagai K, Yamamoto N, Miyashiro I, et al. Multicenter study evaluating the clinical performance of the OSNA assay for the molecular detection of lymph node metastases in gastric cancer patients. Gastric Cancer. 2014;17(2):273-80.

19. Yaguchi Y, Sugasawa H, Tsujimoto H, et al. One-step nucleic acid amplification (OSNA) for the application of sentinel node concept in gastric cancer. Ann Surg Oncol. 2011;18(8):2289-96.

20. Goda H, Nakashiro K, Oka R, et al. One-step nucleic acid amplification for detecting lymph node metastasis of head and neck squamous cell carcinoma. Oral Oncol. 2012;48(10):958-63.
21. Matsuzuka T, Takahashi K, Kawakita D, et al. Intraoperative molecular assessment for lymph node metastasis in head and neck squamous cell carcinoma using one-step nucleic acid amplification (OSNA) assay. Ann Surg Oncol. 2012;19(12):3865-70.

22. Suzuki M, Matsuzuka T, Hashimoto Y, Ikeda M, Saijo S, Omori K. Diagnostic potential of 1-step nucleic acid amplification assay in patients with head and neck squamous cell carcinoma based on CK19 expression in a primary lesion. Head Neck. 2014;38:E239-45.

23. Shinohara $M$, Harada $T$, Nakamura $S$, Oka $M$, Tashiro $H$. Heterotopic salivary gland tissue in lymph nodes of the cervical region. Int J Oral Maxillofac Surg. 1992;21(3):166-71.

24. Leon X, Sancho FJ, Garcia J, Sanudo JR, Orus C, Quer M. Incidence and significance of clinically unsuspected thyroid tissue in lymph nodes found during neck dissection in head and neck carcinoma patients. Laryngoscope. 2005;115(3):470-4.

25. Takahashi K, Nakajima K, Shino M, Toyoda M, Takayasu Y, Chikamatsu K. [Prediction of postoperative lymph node metastasis with a molecular biological test in head and neck cancer]. Nihon Jibiinkoka Gakkai kaiho. 2015;118(2):135-9. 